\title{
Armoring of the Wendelstein 7-X divertor-observation immersion-tubes based on NBI fast-ion simulations
}

\author{
Simppa Äkäslompolo ${ }^{\mathrm{a}, *}$, Peter Drewelow ${ }^{\mathrm{a}}$, Yu Gao ${ }^{\mathrm{b}}$, Adnan Alia ${ }^{\mathrm{a}}$, Sergey Bozhenkov ${ }^{\mathrm{a}}$, Joris Fellinger ${ }^{\mathrm{a}}$, Joachim Geiger ${ }^{\mathrm{a}}$, Dirk \\ Hartmann $^{\mathrm{a}}$, Dag Hathiramani ${ }^{\mathrm{a}}$, Marcin Jakubowski ${ }^{\mathrm{a}}$, Paul McNeely ${ }^{\mathrm{a}}$, Stefan Mohr ${ }^{\mathrm{a}}$, Holger Niemann ${ }^{\mathrm{a}}$, Fabio Pisano ${ }^{\mathrm{c}}$, Norbert \\ Rust $^{\mathrm{a}}$, Aleix Puig Sitjes ${ }^{\mathrm{a}}$, Marcin Sleczka ${ }^{\mathrm{d}}$, Robert Wolf ${ }^{\mathrm{a}}$, W7-X Team \\ ${ }^{a}$ Max-Planck-Institut für Plasmaphysik, Teilinstitut Greifswald, Wendelsteinstrae 1, D-17491 Greifswald \\ ${ }^{b}$ Forschungszentrum Jülich, IEK-4, Jülich, Germany \\ ${ }^{c}$ University of Cagliari, Cagliari, Italy \\ ${ }^{d}$ University of Szczecin, Szczecin, Poland
}

\begin{abstract}
The first neutral beam injector (NBI) experiments of the Wendelstein 7-X stellarator took place in summer 2018. The modelling of the fast ion production and slowing down processes predicts losses of the NBI fast ions to the first wall on the order of $15 \%$. One location receiving a high load (possibly peaking at several $\mathrm{MW} / \mathrm{m}^{2}$ ) is the immersion tube for optical and infrared monitoring of the divertor targets. The stainless steel face of the tube has three vacuum windows, which are sensitive to temperature gradients and overheating. To protect the windows from damage caused by the fast ions, different heat load mitigation techniques were investigated. Given the available time and resources until the first NBI experiments, a protective stainless steel collar mounted at the front of the immersion tubes was regarded the most realistic solution. This contribution describes the fast ion modelling of the loads, the new design, thermal modelling of the design, and finally experimental experience with the protective collar showing heat loads in excess of $1.5 \mathrm{MW} / \mathrm{m}^{2}$. The fast ion heat loads have been assessed computationally with the ASCOT code and experimentally with thermal imaging.
\end{abstract}

Keywords: Wendelstein 7-X, Neutral beam injection, fast ions, plasma facing components, simulation, thermography

\section{Introduction}

The Wendelstein 7-X (W7-X) stellarator has modular superconducting coils and the field is optimized i.a. for improved fast ion confinement. It has been in operation since 2015 , and the first neutral beam injection (NBI) experiments took place in summer 2018. The machine has quite radial NBI injection geometry [1] with initial NBI particle pitch-angles only $17^{\circ}$ or $27^{\circ}$ from perpendicular. This leads to significant NBI fast ion losses [2] to the plasma facing components (PFCs) [3]. The most critical of the loaded components was found to be a set of immersion tubes holding cameras for divertor observations [3, 4].

The immersion tubes are part of the main vacuum barrier, as the cameras are in air and look at the plasma through three thin vacuum windows. The immersion tubes are in outboard side ports, that have identifiers consisting of the letters "AEF", followed by two digits depicting the toroidal section. Thus, the tubes are located in a local magnetic well ("ripple well") between the main coils. Furthermore, the tubes slightly protrude towards the plasma from the surrounding PFCs. Therefore, certain class of ripple trapped fast ions drift to the immersion tube and produce local heat loads of several $\mathrm{MW} / \mathrm{m}^{2}$.

The stainless steel immersion tubes are cooled by compressed air and the system is designed to remove the heating by

*simppa.akaslompolo@alumni.aalto.fi https://orcid.org/0000-0002-9554-5147

Preprint submitted to Fusion Engineering and Design (SOFT 2018, P2.130) plasma radiation (typical design value would be $150 \mathrm{~kW} / \mathrm{m}^{2}$ ) as well as the heating from the cameras inside the tube. The immersion tubes were designed to only be used in the first campaigns of W7-X. A future steady state compatible endoscope will use water cooling and will not have fragile vacuum windows.

A number of methods to alleviate the fast ion heating problem were explored. In 2017, the solution was expected to be optimization of magnetic field of W7-X by utilizing the flexible set of 7 different shapes of main field coils. Certain coil current combinations (magnetic configurations) seemed to remove the fast ion loading from the immersion tubes. However, in December 2017 it was discovered that such currents would induce intolerable electromechanical loads to the coils [5].

Thereafter, different alternative solutions were quickly explored: retracting the immersion tube away from the plasma, welding the most endangered windows shut, or adding a protective collar around the immersion tube face. Only the last solution was realistic in the very short time before the vacuum vessel was closed at the end of March 2018 for the next campaign.

This contribution describes the final design of the protective collar and first experimental results with fast ion heating. The paper starts with section 2 describing the simulation results of the problem, then the implemented collar is described in section 3 . Sections 4 and 5 describe the simulated fast ion and 
thermal plasma heat loads with the collar, respectively. Section 6 introduces the simple thermal model used to calculate heat fluxes from the experimental surface temperatures reported in section 7. A summary concludes the contribution.

\section{Fast ion loading without armoring}

This section describes the original issue of fast ion heating of the immersion tube. The problem was first predicted in detailed modelling: the immersion tube model was included in the configuration scan simulations performed in [3] with the ASCOT suite of codes [6,7]. The codes model the NBI injection geometry, neutral beam ionization, orbit following, fast ion diffusion, slowing down and wall collisions.

The results indicate that there is strong heating of the upper corners of the upper immersion tubes (figure 1) for the normal magnetic field direction. In these simulations, two sources from both of the NBI injectors were injecting $1.7 \mathrm{MW}$ of hydrogen atoms with up to $55 \mathrm{keV}$ energy.

A modified magnetic field configuration, dubbed "Inward shifted high mirror", would have moved the load to the carbon tiles, but this configuration is incompatible with the mechanical properties of the superconducting coils.

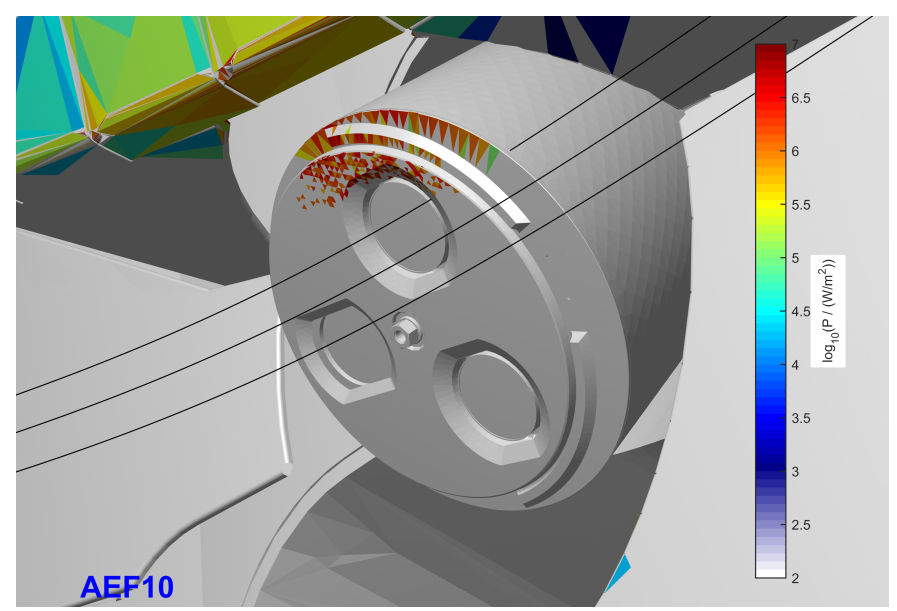

Figure 1: The NBI fast ion heat load onto the AEF10-port immersion tube and adjacent PFCs [8]. The fast ions are trapped in a local magnetic well and drift upward. Some of them hit the corner of the immersion tube or the carbon tiles above it. In front of the immersion tube there is a circular shutter plate that can be rotated to shield the vacuum windows during glow discharge cleaning. This calculation is for the high mirror configuration, which has generally the most benign fast ion loads of all of the reference magnetic configurations. The fast ion immersion tube issue persists in other configurations as well. The three black lines are field lines separated vertically by $3 \mathrm{~cm}$.

\section{The implemented armor}

Figure 2 shows the final design for the protective collar. This design was reached after first using simple shapes to find the optimal dimensions for the component. The collar extends $\sim 4 \mathrm{~cm}$ above the face of the immersion tube, which could not be extended without limiting the field of view of the camera. The gap in the collar is in an unloaded part which would obscure key areas of interest for the thermal camera next to it.

The material was chosen to be the 1.4429 stainless steel. A carbon collar was rejected due to longer manufacturing time and higher cost. Each collar is in view of the thermal camera in the opposed immersion tube, so infrared monitoring is in principle possible. However, thermal imaging of polished metal surfaces is difficult due to low emissivity and high reflectivity. Thus, three surface finishing methods were tested: diamond coating, chemical etching and glass bead blasting. The last was chosen and resulted in surface emissivity of $0.74-0.77$ at the spectral range $8-10 \mu \mathrm{m}$, which is the sensitivity range of the IR camera. The collar was fastened to the immersion tube with two M4 screws that fit to existing screw holes.
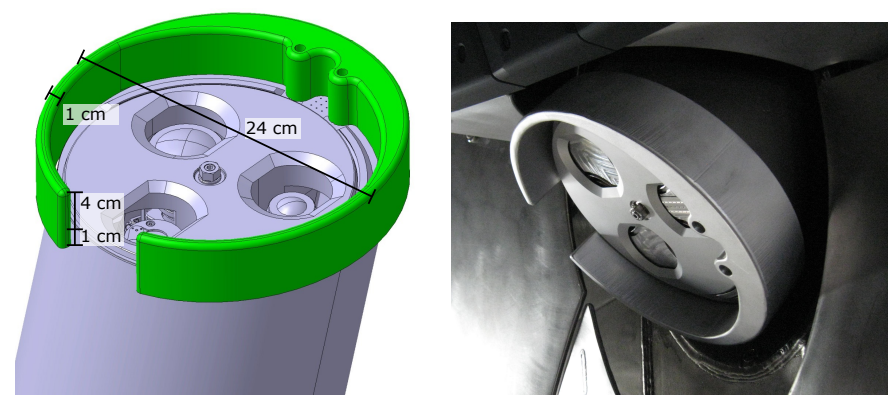

Figure 2: The stainless steel protective collar. The final design (left panel) and as installed to the port AEF20. The rotatable shutter is open in the left panel and closed in the right panel.

\section{Fast ion loading with the armoring}

The final collar geometry was imported to ASCOT and the fast ion load was calculated. It was found that the collar stops the fast ions from reaching the immersion tube face, as illustrated in figure 3. The collar fast ion load can reach up to several $\mathrm{MW} / \mathrm{m}^{2}$ in the highest loading configuration expected for 2018 experiments (the so called "high iota" configuration).

The simulations were performed with magnetic fields with various plasma pressure to magnetic pressure ratios $(\beta \mathrm{s})$. When the average beta $\langle\beta\rangle$ is increased, a small but rising number of very low parallel velocity fast ions may reach the face of the immersion tube. Plasmas with $\langle\beta\rangle>1 \%$ are currently rarely achieved in W7-X.

\section{Thermal plasma loading}

The original immersion tube protrudes slightly from the surrounding PFCs, so extending the tube by further $4 \mathrm{~cm}$ may bring it into contact with thermal plasma. This was studied by moving the immersion tube model with a $4 \mathrm{~cm}$ thick collar closer to the plasma and performing field line diffusion calculations [9] to assess the heat load in different magnetic configurations and different plasma $\langle\beta\rangle_{\mathrm{s}}$. In these calculations the plasma energy is modelled with markers, that flow out along the magnetic field lines and diffuse perpendicular to it. Figure 4 shows that for most studied configurations a collar thickness of $4 \mathrm{~cm}$ will not 


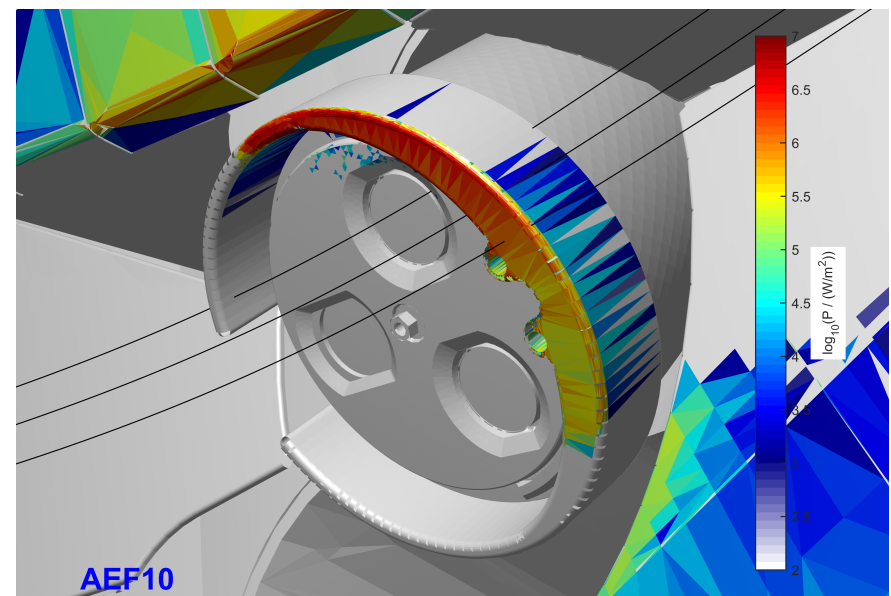

Figure 3: The fast ion loads with the final design of the protective collar. The magnetic configuration is "high iota" with $\langle\beta\rangle=0 \%$ and NBI sources 7 and 8 injecting. The black lines are magnetic field lines.

result in thermal plasma interacting with the collar. However, high $\langle\beta\rangle$, "low shear" or "outward shifted" magnetic configurations may result in thermal plasma to heat the collar. These $\langle\beta\rangle$ values and configurations were not be present in 2018 experiments and no indication of direct thermal plasma heating of the collars was found. The parameters for the diffusion calculations were set to velocity $v=310 \mathrm{~km} / \mathrm{s}$ and the perpendicular diffusion coefficient $D_{\perp}=1 \mathrm{~m}^{2} / \mathrm{s}$ and the thermal power to $8 \mathrm{MW}$.

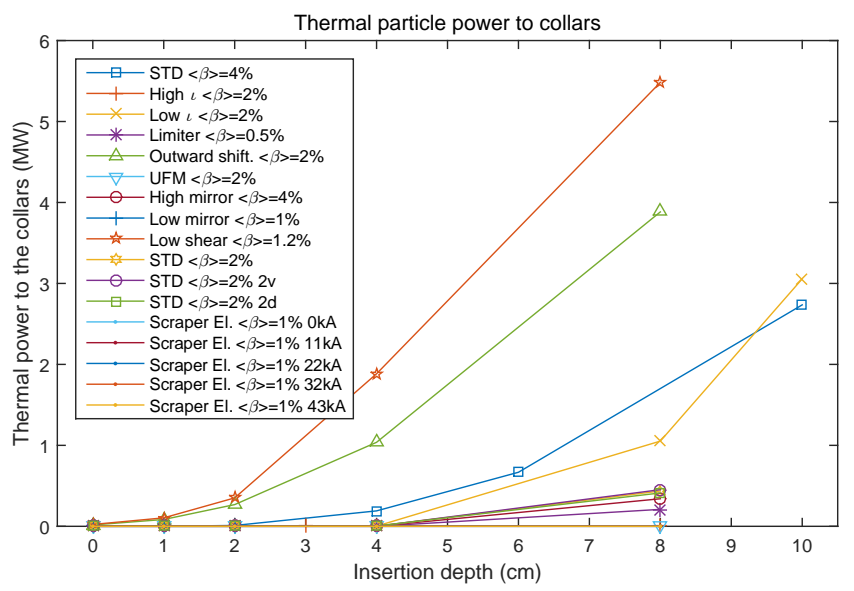

Figure 4: The heat load due to thermal plasma according to field line diffusion calculations. The abscissa "insertion depth" depicts moving the immersion tube towards the plasma from the implemented set-up $(0 \mathrm{~cm})$. The various lines are different magnetic configuration variations. The most critical analyzed configurations, high $\langle\beta\rangle$, "low shear" and "outward shifted", were not present in the 2018 experiments.

\section{Thermal modelling}

In the case of fast ion heating of the collar, the dominating heat source is the fast ion heat flux arriving to the surface of the collar. The temperatures are too low for radiation losses to be significant. The temperature behaviour is dominated by heat conduction into the collar.
The change of surface temperature $\Delta T$ of a semi-infinite solid due to a constant heat flux $q\left(\mathrm{~W} / \mathrm{m}^{2}\right)$ as a function of time $t$ is given by the equation $([\overline{10]})$

$$
\Delta T(t)=q \frac{2}{\sqrt{\pi \lambda \rho c_{p}}} \sqrt{t}
$$

where the constants for the collar steel are approximately the following: heat conductivity $\lambda=15 \mathrm{~W} /(\mathrm{K} \mathrm{m})$; density $\rho=7900 \mathrm{~kg} / \mathrm{m}^{3}$; specific heat $c_{p}=470 \mathrm{~J} /(\mathrm{kg} \mathrm{K})$. The assumption of semi-infinity is valid, as can be checked by considering the penetration of the heat in the solid [10]:

$$
\Delta T(t, x)=\frac{q x}{\lambda}\left(\frac{1}{\sqrt{\pi} F} \exp \left(-F^{2}\right)-\operatorname{erfc}(F)\right),
$$

where $F=\sqrt{\frac{\rho c_{p}}{4 \lambda}} \frac{x}{\sqrt{t}}$ and $x$ is distance from the heated surface. The penetration of the heat through the $4 \mathrm{~cm}$ of steel takes several seconds.

\section{Experimental results}

In August 2018, the first injections of NBI power to W7-X plasma took place. The length of NBI injection was gradually increased while continuously monitoring the plasma and plasma facing components for signs of NBI fast ion loading. The main and most successful tool for this were the thermal cameras in the AEF-port immersion tubes. The thermal imaging confirmed the predicted fast ion hot spot locations. (Quantitative comparison is beyond the scope of the current contribution.)

Figure 5 shows a thermal image from a plasma with NBI injection. In that particular experiment, the fast ions heat up the collar surface by $\sim 40 \mathrm{~K}$ in $300 \mathrm{~ms}$. The measured temperature may be an underestimate due to sub-pixel details being blurred. A more detailed analysis will be presented in a future paper. However, current estimate gives heat loads in excess of $1.5 \mathrm{MW} / \mathrm{m}^{2}$, as interpreted through equation (1). Nevertheless, visual post-mortem analysis of the collars revealed no markings attributable to fast ion heating. While the heating is of no concern to the steel collar, the windows are specified to handle temperature changes of only $25 \mathrm{~K} / \mathrm{min}$ due to the sensitive soldered joint between the window and the frame. The manufacturer (Lewvac) specifies maximum temperatures of $450{ }^{\circ} \mathrm{C}$ and $200^{\circ} \mathrm{C}$, for the sapphire and zinc-selenide windows, respectively.

\section{Summary}

This paper summarizes the outcome of armoring the AEF-port immersion tubes against fast ion impacts in the Wendelstein 7$\mathrm{X}$ stellarator. The problem of NBI heatloads was identified in fast ion simulations. The fragile vacuum windows near a fast ion hot-spot were protected by installing a protective stainless steel collar in front of each immersion tube. The design was evaluated with further fast ion and field line diffusion calculations. First experimental measurements of NBI fast ion loads 


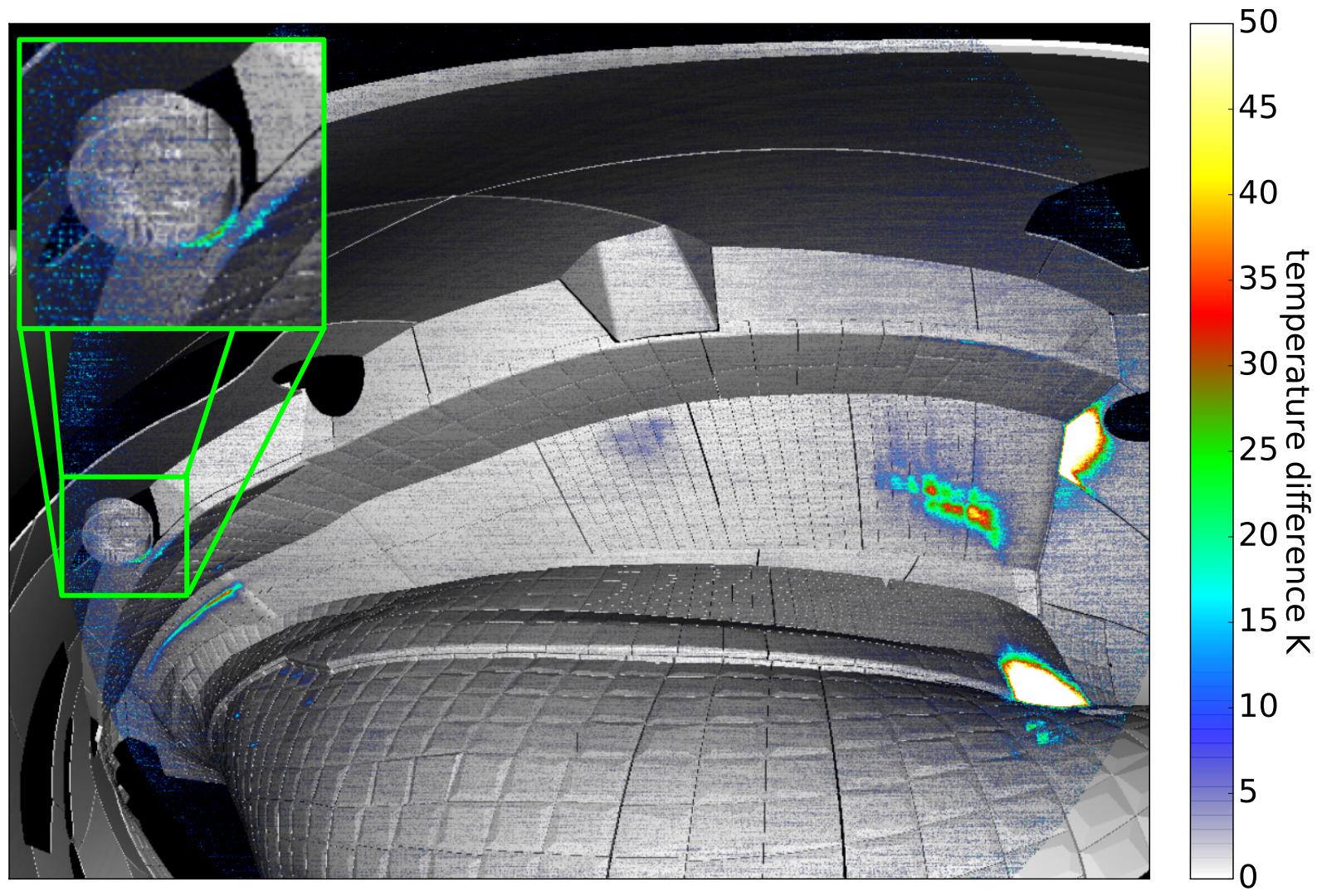

Figure 5: Thermal image showing the divertor area during NBI heating. The insert displays the immersion tube and the adjacent carbon tiles. A frame before the NBI started was subtracted to highlight the heating due to NBI. The collar was at $134^{\circ} \mathrm{C}$ and the baffle at $137^{\circ} \mathrm{C}$ when the NBI heating commenced. The camera noise level is approximately $3^{\circ} \mathrm{C}$. The other hot spots in the image include further fast ion loss hot spots as well as NBI shinethrough to the beamdump and reflections of them. The image is from program 20180822.017@6.79s from camera AEF21.

show significant heating of the collar surfaces and indicate the project was justified.

Outstanding issues include simulation results indicating that operation with high plasma beta may cause certain fast ions to bypass the collar and land on the immersion tube or cause thermal plasma to heat the collars. Similarly, certain magnetic field configurations could cause thermal plasma to heat the collar.

\section{Acknowledgements}

The authors would like to thank all the participants of the CCB246 meetings on the fast ion heating issue. The work was partly funded by Walter Ahlström foundation. This work has been carried out within the framework of the EUROfusion Consortium and has received funding from the Euratom research and training programme 2014-2018 and 2019-2020 under grant agreement No 633053. The views and opinions expressed herein do not necessarily reflect those of the European Commission.

\section{References}

[1] N. Rust, B. Heinemann, B. Mendelevitch, A. Peacock, M. Smirnow, W7$x$ neutral-beam-injection: Selection of the NBI source positions for experiment start-up, Fusion Engineering and Design 86 (68) (2011) 728 - 731, proceedings of the 26th Symposium of Fusion Technology (SOFT-26). doi:10.1016/j.fusengdes.2011.03.054
[2] M. Drevlak, J. Geiger, P. Helander, Y. Turkin, Fast particle confinement with optimized coil currents in the w7-x stellarator, Nuclear Fusion 54 (7) (2014) 073002. doi:10.1088/0029-5515/54/7/073002

[3] S. Äkäslompolo, M. Drevlak, Y. Turkin, S. Bozhenkov, T. Jesche, J. Kontula, T. Kurki-Suonio, R. W. and, Modelling of NBI ion wall loads in the w7-x stellarator, Nuclear Fusion 58 (8) (2018) 082010. doi: 10.1088/1741-4326/aac4e5

[4] J. Baldzun, Fast ion orbit losses in w7-x, Tech. Rep. PLM:1-CDY-T0005, Max-Planck-Institute for Plasma Physics, (Internal report) (2012).

[5] J. Zhu, V. Bykov, Overview of op1.2b regimes results from gm6.03, Tech. Rep. PLM:1-GXA10M-T0122, Max-Planck-Institute for Plasma Physics, (Internal report) (2018).

[6] E. Hirvijoki, O. Asunta, T. Koskela, T. Kurki-Suonio, J. Miettunen, S. Sipilä, A. Snicker, S. Äkäslompolo, ASCOT: Solving the kinetic equation of minority particle species in tokamak plasmas, Computer Physics Communications 185 (4) (2014) 1310-1321. arXiv: 1308.1904, doi: $10.1016 / j . c p c .2014 .01 .014$

[7] O. Asunta, J. Govenius, R. Budny, M. Gorelenkova, G. Tardini, T. KurkiSuonio, A. Salmi, S. Sipilä, the ASDEX Upgrade Team, the JET EFDA Contributors, Modelling neutral beams in fusion devices: Beamletbased model for fast particle simulations, Computer Physics Communications 188 (2015) 33-46. doi:10.1016/j.cpc.2014.10.024

[8] S. Äkäslompolo, Magnetic configuration for minimizing nbi wall load to steel components of $\mathrm{w} 7-\mathrm{x}$ in op1.2, W7-X weekly physics program meeting 15.02.2017, PLM: 1-CDY-Y0006.

[9] S. Bozhenkov, J. Geiger, M. Grahl, J. Kißlinger, A. Werner, R. Wolf, Service oriented architecture for scientific analysis at w7-x. an example of a field line tracer, Fusion Engineering and Design 88 (11) (2013) 2997 3006. doi:10.1016/j.fusengdes.2013.07.003

[10] H. S. Carslaw, J. C. Jaeger, Conduction of Heat in Solids, Oxford University Press, 1986. 